\title{
Equações hipsométricas, volumétricas e funções de afilamento para Pinus spp.
}

\author{
Hypsometric and volumetric equations and taper functions for Pinus spp.
}
Marcos Felipe Nicoletti ${ }^{1 *}$, Luciano Lambert ${ }^{2}$, Philipe Ricardo Caserimo Soares ${ }^{1}$, Girlene da Silva $\mathrm{Cruz}^{1}$, Bruno Rafael Silva Almeida ${ }^{1}$, Thiago Floriani Stepka ${ }^{1}$

${ }^{1}$ Universidade do Estado de Santa Catarina, Lages, SC, Brasil. * Autor para correspondência: marcos.nicoletti@udesc.br

${ }^{2}$ Klabin S/A, Otacílio Costa, SC, Brasil.

Submissão: 29/10/2018 / Aceite: 02/09/2020

\begin{abstract}
RESUMO
O emprego de ferramentas como a modelagem florestal torna-se cada vez mais importantes no planejamento florestal pois geram estimativas acuradas da produção dos povoamentos. O objetivo do estudo foi realizar o ajuste de modelos hipsométricos, volumétricos e de afilamento para quatro espécies de Pinus na Região Serrana de Santa Catarina. As espécies utilizadas foram Pinus taeda, Pinus elliottii, Pinus greggii e Pinus patula todas implantadas e conduzidas sobre o mesmo plano de manejo florestal. $O$ povoamento está localizado no município de Correia Pinto, Santa Catarina, com 14 anos de idade. Para as análises foram ajustados modelos matemáticos com base nos dados do censo florestal. Para a escolha do melhor modelo utilizaram-se os critérios estatísticos: o coeficiente de determinação ajustado $\left(R^{2}{ }^{2}\right.$ ), erro padrão da estimativa $\left(S_{y \times \%}\right)$, análise gráfica dos resíduos. As equações hipsométricas apresentaram $R^{2}$ ajustado relativamente baixos, com valores variando entre 0,14 e 0,35 para as espécies e $S_{y x} \%$ variando entre $7,67 \%$ a $4,86 \%$. Em relação as equações volumétricas, o $R^{2}$ ajustado ficou acima de 0,91 e o $S_{y \times \%}$ foi inferior a $10 \%$. No que diz respeito as funções de afilamento o melhor modelo para descrever o perfil do fuste das espécies foi Hradetzky, com $\mathrm{R}^{2}$ ajustado acima de 0,96 e $\mathrm{S}_{\mathrm{yx}}$ inferior a $10 \%$. Os modelos de Naslund, Spurr I e II e Naslund modificado foram selecionados para estimar a volumetria das espécies e; o modelo de afilamento de Hradetzky, foi superior aos demais, sendo selecionado para descrever o perfil do fuste das espécies na área de estudo.
\end{abstract}

PALAVRAS-CHAVE: cubagem, manejo florestal, perfil do fuste.

\section{ABSTRACT}

The use of tools such as forest modeling becomes increasingly important in forest planning as they generate accurate estimates of the stands' production. The aim of this study was to adjust the hypsometric, volumetric, and taper models for four Pinus species in the Mountain region of Santa Catarina in Brazil. The species Pinus taeda, Pinus elliottii, Pinus greggii, and Pinus patula were all planted and maintained under the same forest management plan. The 14-year-old stand is located in the municipality of Correia Pinto, Santa Catarina, Brazil. For the analyses, mathematical models were adjusted based on data from the forest census. The following statistical criteria were used to choose the most appropriate model: the adjusted coefficient of determination $\left(\mathrm{R}^{2}{ }_{\mathrm{adj}}\right)$, standard error $\left(\mathrm{S}_{\mathrm{y} . \mathrm{x} \%}\right)$ and graphic residual analysis. The hypsometric equations presented relatively low values for the adjusted $R^{2}$, ranging from 0.14 to 0.35 for the species, while the values for $S_{y . x \%}$ ranged between $7.67 \%$ and $4.86 \%$. As for the volumetric equations, the adjusted $\mathrm{R}^{2}$ was above 0.91 , and $\mathrm{S}_{\mathrm{y} . \mathrm{x} \%}$ was under $10 \%$. With regard to the taper functions, the Hradetzky model most appropriately described the stem profile of the species, with values above 0.96

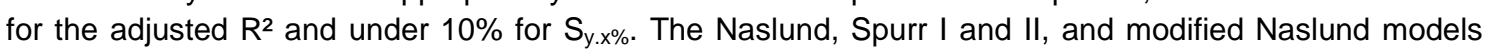
were selected to estimate the species' volumetry. The Hradetzky taper model was found to be superior to the others and was thus selected to determine the stem profile of the species in the field research area.

KEYWORDS: volumetric measurements, forest management, stem profile.

\section{INTRODUÇÃO}

No Brasil, a área de florestas plantadas totalizou 7,83 milhões de hectares em 2018, o que corresponde a aproximadamente $1 \%$ do território brasileiro. Destes, os plantios com espécies do gênero 
Eucalyptus spp. ocupam 5,7 milhões de hectares ( $72 \%$ do total) distribuídos na sua maioria no sudeste do país. Já os plantios de Pinus representam 1,6 milhões de hectares ( $20 \%$ do total) e concentram-se, principalmente, no Paraná (42\%) e em Santa Catarina (34\%) (IBA 2019).

Dentre os gêneros mais plantados, o Pinus veio como uma alternativa ao esgotamento da madeira de araucária, espécie nativa do Sul do país. O uso do Pinus representa, uma forma de minimizar a exploração de florestas nativas, constituindo-se em uma alternativa para gerar matéria prima florestal de forma renovável e sustentável (COELHO 2010).

O emprego de ferramentas para atividades no planejamento florestal se torna de grande interesse ao setor florestal, assim alcançando informações referentes a quantificação e a previsão de suas florestas (ABREU 2000). A modelagem florestal torna-se cada vez mais importantes para gerar estimativas acuradas da produção dos povoamentos florestais. O emprego de equações hipsométricas, volumétricas e de afilamento são imprescindíveis no inventário florestal, pois permitem estimativas mais precisas referentes a volumetria da área a ser manejada (SOARES et al. 2011, NICOLETTI 2017).

No setor florestal a utilização de equações hipsométricas, faz com que ocorra a diminuição no tempo de medição das variáveis dendrométricas, tornando assim os inventários mais rápidos, econômicos e precisos, visto que essas equações estimam as alturas através da relação altura/diâmetro (THIERSCH et al. 2013, ATANAZIO et al. 2017).

O emprego de equações de volume para a estimativa da volumetria da área a ser colhida, faz com que as empresas tomem decisões mais precisas referente as análises de investimentos e volumetria a ser retirada a cada ano (RUFINI et al. 2010). A estimativa de volume total ou comercial são variáveis de grande interesse a serem estimadas em florestas sujeitas à prática do manejo florestal. Estimando-se estes volumes com precisão, os planos de manejo, as análises de investimentos e as tomadas de decisão também serão precisos (RUFINI et al. 2010, STEPKA et al. 2017).

As equações de afilamento são tidas como ferramentas importantes uma vez que através delas se obtêm estimativas de volume mais próximas do real, pois permitem estimar o volume em qualquer segmento ao longo do fuste, maximizando assim o aproveitamento do povoamento florestal (GARCIA et al. 1993, MACHADO et al. 2002, LEITE et al. 2006, COSTA et al. 2016).

Diante disto, o presente estudo teve por objetivo ajustar equações hipsométricas, volumétricas e de afilamento para quatro espécies de Pinus na Região Serrana de Santa Catarina.

\section{MATERIAL E MÉTODOS}

\section{Área de estudo}

A pesquisa foi realizada na Fazenda Bandeirinhas, município de Correia Pinto, Estado de Santa Catarina (SC), no Sul do Brasil, a cerca de 950 metros de altitude média (Lat. $27^{\circ} 44,126^{\prime \prime} \mathrm{S}$, Log. $50^{\circ}$ 21,873" W). Conforme a classificação de Köppen, o clima da área de estudo é do tipo Cfb, mesotérmico, subtropical úmido, com invernos frios e verões quentes e úmidos (ALVARES et al. 2013). A precipitação anual média de $1.516 \mathrm{~mm}$ e a temperatura média anual de 16,4 으 (AGUIAR et al. 2011). O solo de maior representatividade do local é o Cambissolo Húmico, com associações de Cambissolo Háplicos e Neossolo Litólico nas áreas mais declivosas (AGUIAR et al. 2011).

\section{Coleta de dados}

No censo florestal da área, foram coletadas o diâmetro à altura do peito (DAP) com utilização de suta e a altura total $\left(h_{t}\right)$ dos indivíduos com Hipsômetro Blume Leiss. As alturas foram mensuradas na primeira linha de plantio e $20 \%$ das árvores de diâmetro dominante para cada espécie. Segundo THOMAS et al. (2006), também conhecida como altura dominante $h_{100}$. O seu uso justifica-se por ser pouco influenciada pelos desbastes por baixo e por possuir alta correlação com a produção total do povoamento. $\mathrm{Na}$ sequência, realizou-se a cubagem rigorosa utilizando o método de Smalian de 75 árvores, sendo 20 árvores de Pinus taeda L., 17 árvores Pinus elliottii Engelm var. elliotti, 18 árvores de Pinus greggii Engelm e 20 árvores de Pinus patula Schlechtd. \& Cham.

\section{Análise de dados}

\section{Relação Hipsométrica}

Para o ajuste da relação hipsométrica, utilizaram-se dados de altura total e diâmetro à altura do peito obtidos no censo florestal e na cubagem. Os modelos matemáticos ajustados neste trabalho foram selecionados na literatura e estão descritos na Tabela 1.

Modelos volumétricos

Com os dados obtidos na cubagem, os volumes individuais para cada uma das árvores-amostra foram calculados. Os dados destas árvores serviram de base para ajuste dos modelos de volume 
relacionados na Tabela 2. O estudo dos modelos volumétricos possibilitou, por meio de métodos estatísticos, definir o modelo que melhor estime o volume para cada espécie do plantio estudado.

Tabela 1. Modelos de relação hipsométrica testados para quatro espécies do gênero Pinus.

Table 1. Hypsometric relation models tested for four species of the genus Pinus.

\begin{tabular}{|c|c|c|}
\hline № & MODELO & AUTOR \\
\hline 1 & $\ln \left(\mathrm{h}_{\mathrm{t}}\right)=\beta_{0}+\beta_{1} \frac{1}{\mathrm{dap}}+\varepsilon_{\mathrm{j}}$ & Curtis \\
\hline 2 & $\underset{1}{\ln \left(\mathrm{h}_{6}\right)}=\beta_{0}+\beta_{1} \ln (\mathrm{dap})+\varepsilon_{1}$ & Stoffels \\
\hline 3 & $\overline{\mathrm{h}_{\mathrm{t}}-1,3}=\beta_{0}+\beta_{1} \frac{-}{\mathrm{dap}}+\varepsilon_{\mathrm{i}}$ & Petterson \\
\hline 4 & $\mathrm{~h}_{t}-1,3=\frac{\mathrm{dap}}{\beta_{0}+\beta_{1} \mathrm{dap}^{2}}+\varepsilon_{\mathrm{i}}$ & Naslund \\
\hline 5 & $\frac{\mathrm{d}^{2}}{\mathrm{~h}_{t}}=\beta_{0}+\beta_{1} \mathrm{dap}+\beta_{2} \mathrm{dap}^{2}+\varepsilon_{\mathrm{i}}$ & Prodan \\
\hline 6 & $\mathrm{~h}_{\mathrm{t}}=\beta_{\mathrm{o}}+\beta_{1} \ln \mathrm{dap}+\varepsilon_{\mathrm{i}}$ & Henricksen \\
\hline 7 & $\mathrm{~h}_{\mathrm{t}}=\beta_{\mathrm{o}}+\beta_{1} \frac{1}{\mathrm{dap}}+\varepsilon_{\mathrm{i}}$ & Assmann \\
\hline 8 & $\mathrm{~h}_{t}=\beta_{0}+\beta_{1} \mathrm{dap}+\beta_{2} \operatorname{dap}^{2}+\varepsilon_{\mathrm{i}}$ & Trorey \\
\hline 9 & $\mathrm{~h}_{t}=\beta_{0}+\beta_{1} \operatorname{dap}+\varepsilon_{\mathrm{dap}^{2}}$ & Linear Simples \\
\hline 10 & $\mathrm{~h}_{t}-1,3=\frac{1}{\beta_{0}+\beta_{1} \operatorname{dap}+\beta_{1} \mathrm{dap}^{2}}+\varepsilon_{\mathrm{i}}$ & Prodan 2 \\
\hline 11 & $\ln h_{t}=\beta_{0}+\beta_{1} \operatorname{dap}+\beta_{2} \operatorname{dap}^{2}+\varepsilon_{\mathrm{i}}$ & AD1 \\
\hline 12 & $\ln h_{t}=\beta_{0}+\beta_{1} \operatorname{dap}^{2}+\varepsilon_{\mathrm{i}}$ & AD2 \\
\hline
\end{tabular}

Em que: $\ln =$ logaritmo neperiano; dap = diâmetro medido à $1,3 \mathrm{~m}$ do solo $(\mathrm{cm}) ; h_{t}=$ altura total $(\mathrm{m}) ; \beta_{\mathrm{i}}=$ parâmetros a serem estimados e $\varepsilon_{i}=$ erro aleatório.

Tabela 2. Modelos volumétricos testados para quatro espécies do gênero Pinus.

Table 2. Volumetric models tested for four species of the genus Pinus.

\begin{tabular}{|c|c|c|}
\hline № & MODELO & AUTOR \\
\hline 1 & $v_{i}=\beta_{0}+\beta_{1}\left(\operatorname{dap}^{2} h_{t}\right)+\varepsilon_{\mathrm{i}}$ & Spurr I \\
\hline 2 & $v_{i}=\beta_{0}+\beta_{1} \mathrm{dap}+\varepsilon_{\mathrm{i}}$ & Burkhault I \\
\hline 3 & $v_{i}=\beta_{0}+\beta_{1}\left(\operatorname{dap} h_{2}\right)+\varepsilon_{i}$ & Spurr II \\
\hline & $v_{i}=\beta_{0}+\beta_{1} \mathrm{dap}+\beta_{2} \mathrm{dap}^{2} \varepsilon_{1}$ & Honenald-Krenn \\
\hline & $v_{i}=\beta_{0}+\beta_{1}\left(\frac{1}{\operatorname{dap}}\right)+\beta_{2}\left(\frac{1}{\operatorname{dap}}\right)^{-}+\varepsilon_{\mathrm{j}}$ & Bonetes I \\
\hline 6 & $v_{i}=\beta_{0}+\beta_{1}\left(\operatorname{dap}^{2} h_{t}\right)+\beta_{2}\left(\frac{1}{\sqrt{\operatorname{dap}}}\right)+\varepsilon_{\mathrm{i}}$ & Omerod MII \\
\hline 7 & $v_{i}=\beta_{0}+\beta_{1}\left(\operatorname{dap}^{2}\right)+\beta_{2}\left(\operatorname{dap}^{2} h_{t}\right)+\beta_{3}\left(\mathrm{~h}_{t}\right)+\varepsilon_{\mathrm{i}}$ & Naslund \\
\hline 8 & $v_{i}=\beta_{0}+\beta_{1}\left(\operatorname{dap}^{2}\right)+\beta_{2} \ln \left(\operatorname{dap}^{2} h_{t}\right)+\beta_{3}\left(\operatorname{dap~h}_{t}^{2}\right)+\beta_{4} h^{2} \varepsilon_{1}$ & Naslund Modificado \\
\hline & $\ln \left(v_{i}\right)=\beta_{0}+\beta_{1} \ln (\mathrm{dap})+\varepsilon_{\mathrm{i}}$ & Husch \\
\hline & $\ln \left(v_{i}\right)=\beta_{0}+\beta_{1} \ln \left(\operatorname{dap}^{2} \mathrm{~h}_{i}\right)+\varepsilon_{\mathrm{i}}$ & Spurr III \\
\hline & $\begin{array}{l}\ln \left(\mathrm{v}_{1}\right)=\beta_{0}+\beta_{1} \ln (\mathrm{dap})+\beta_{2} \ln \left(\mathrm{h}_{t}\right)+\varepsilon_{1} \\
\ln \left(\mathrm{v}_{1}\right)=\beta_{0}+\beta_{1} \ln (\mathrm{dap})+\beta_{2} \ln ^{2}(\mathrm{dap})+\beta_{3} \ln \left(\mathrm{h}_{t}\right)+\beta_{4} \ln ^{2}(\mathrm{dap})+\varepsilon_{1}\end{array}$ & $\begin{array}{l}\text { Schumacher-Hall } \\
\text { Prodan }\end{array}$ \\
\hline
\end{tabular}

Em que: $\mathrm{In}=$ logaritmo neperiano; log = logaritmo; dap = diâmetro medido à $1,3 \mathrm{~m}$ do solo $(\mathrm{cm}) ; h_{t}=$ altura do fuste $(\mathrm{m})$; $\beta_{\mathrm{i}}=$ parâmetros a serem estimados e; $\varepsilon_{\mathrm{i}}=$ erro aleatório.

Funções de afilamento

O ajuste dos modelos não-segmentados foi feito para cada espécie aos 14 anos, com a finalidade de obter equações ajustadas e quantificar o volume total por hectare para cada espécie. Os modelos nãosegmentados testados são descritos na Tabela 3. As potências usadas para o ajuste do modelo de 
potências inteiras e fracionárias foram selecionadas pelo método Stepwise, sendo testadas as seguintes potências: 0,005; 0,09; 0,08; 0,07; 0,06; 0,05; 0,04;0,03;0,02;0,01;0,9;0,8;0,7;0,6;0,5;0,4;0,3;0,2;0,1; $1 ; 2 ; 3 ; 4 ; 5 ; 10 ; 15 ; 20$, e 25 .

Tabela 3. Modelos de afilamento testados para quatro espécies do gênero Pinus.

Table 3. Taper models tested for four species of the genus Pinus.

\begin{tabular}{|c|c|c|}
\hline № & MODELO & AUTOR \\
\hline 1 & $\frac{\mathrm{d}_{i}}{\operatorname{dap}}=\sqrt{\beta_{0}+\beta_{1}\left(\frac{\mathrm{h}_{i}}{\mathrm{~h}_{i}}\right)+\beta_{2}\left(\frac{\mathrm{h}_{i}}{\mathrm{~h}_{\mathrm{t}}}\right)^{2}+\varepsilon_{\mathrm{i}}}$ & $\begin{array}{l}\text { KOZAK et al. } \\
(1969)\end{array}$ \\
\hline 2 & $\frac{\mathrm{d}_{i}}{\operatorname{dap}}=\beta_{0}+\beta_{1}\left(\frac{\mathrm{h}_{i}}{\mathrm{~h}_{\mathrm{z}}}\right)+\beta_{2}\left(\frac{\mathrm{h}_{i}}{\mathrm{~h}_{t}}\right)^{2}+\beta_{3}\left(\frac{\mathrm{h}_{i}}{\mathrm{~h}_{t}}\right)^{3}+\beta_{4}\left(\frac{\mathrm{h}_{i}}{\mathrm{~h}_{\mathrm{t}}}\right)^{4}+\beta_{5}\left(\frac{\mathrm{h}_{i}}{\mathrm{~h}_{\mathrm{t}}}\right)^{5}$ & $\begin{array}{l}\text { SCHÖEPFER } \\
(1966)\end{array}$ \\
\hline 3 & $\frac{\mathrm{d}_{i}}{\operatorname{dap}}=\beta_{0}+\beta_{1}\left(\frac{\mathrm{h}_{i}}{\mathrm{~h}_{\tau}}\right)^{\mathrm{p} 1}+\beta_{2}\left(\frac{\mathrm{h}_{i}}{\mathrm{~h}_{\tau}}\right)^{\mathrm{p}^{2}}+\beta_{\mathrm{n}}\left(\frac{\mathrm{h}_{i}}{\mathrm{~h}_{\tau}}\right)^{\mathrm{pn}}+\varepsilon_{\mathrm{i}}$ & $\begin{array}{l}\text { HRADETZKY } \\
(1976)\end{array}$ \\
\hline
\end{tabular}

Em que: dap = diâmetro medido à 1,3 $\mathrm{m}$ do solo $(\mathrm{cm}) ; d_{i}=$ diâmetro em diferentes alturas $(\mathrm{cm})$ medido em altura $h_{i} ; h_{t}=$ altura do fuste $(m) ; h_{i}=$ altura ao longo do fuste; $\beta_{0}=$ parâmetros a serem estimados; $p n=$ potência selecionada para modelo de Hradetzky; $\varepsilon_{i}=$ erro aleatório.

Para a inclusão ou não de uma variável, níveis críticos para o teste $F$ com $\alpha$ de 0,05 e 0,10 foram utilizados, respectivamente, para a entrada e a saída de uma variável.

Critérios de avaliação e escolha dos modelos

Para a verificação do melhor modelo de regressão, analisou-se o ajuste de cada equação comparativamente, observando-se os critérios estatísticos definidos pelo: a) coeficiente de determinação ajustado, que expressa a quantidade de variação total explicada pela regressão, onde seus resultados variam de 0 a 1 , sendo que mais próximo de 1 melhor será o ajuste da linha da regressão; b) erro padrão da estimativa para verificar a precisão do ajuste, seus resultados variam de 0 a 1 ou $0 \%$ a $100 \%$, sendo que quanto menor for o erro, melhor são as estimativas obtidas na equação; $c$ ) valor de $F$ que quanto maior for melhor será considerado; d) valor ponderado dos escores estatísticos, este parâmetro atribui valores ou pesos aos parâmetros estatísticos. As estatísticas foram ordenadas de acordo com a sua eficiência, sendo atribuído peso 1 para a equação mais eficiente, 2 para a segunda e assim sucessivamente (ranking), conforme metodologia descrita por THIERSCH (1997).

Após essa classificação individual, efetuou-se o somatório da pontuação para cada modelo matemático, sendo que a equação de volume que recebeu a menor soma foi recomendada como mais adequada para uso; e f) distribuição gráfica dos resíduos, a análise de resíduos gráficos é um método que, embora visual, é crucial para a escolha do modelo melhor ajustado. Mostra a dispersão dos valores estimados em relação ao medido. Quanto menor e mais uniforme a dispersão de resíduos, melhor é o ajuste. As avaliações e análises foram realizadas por meio do Software RStudio, versão 1.0.143 utilizando diversos pacotes de análise e Microsoft Excel ${ }^{\circledR} 2013$.

\section{RESULTADOS E DISCUSSÃO}

\section{Relação Hipsométrica}

As equações apresentarem coeficientes de determinação $\left(R_{\text {aj }}^{2}\right)$ relativamente baixos, com o mínimo de 0,144 e máximo de 0,355 , erro padrão de estimativa $\left(S_{\mathrm{yx}}\right)$ variando de $1,1 \mathrm{~m}$ a $1,4 \mathrm{~m}$ e erro padrão de estimativa percentual $\left(S_{\mathrm{yx}} \%\right.$ ) variando de $7,67 \%$ a $4,86 \%$ (Tabela 4 ).

Os baixos valores dos coeficientes de determinação ajustados $\left(R^{2}{ }_{a j}\right)$ são comuns para equações hipsométricas devido à baixa correlação entre altura e diâmetro (BARROS et al. 2002). Quando comparados os resultados do coeficiente de determinação ajustado presente em demais estudos de relação hipsométrica disponibilizados na literatura, nota-se que os valores variam respectivamente de 0,34 a 0,54 (BARROS et al. 2002, COSTA 2008, DONADONI et al. 2010, ARAÚJO et al. 2012).

No entanto os baixos valores do erro padrão da estimativa $\left(S_{y . x \%}\right)$ mostram que as equações estimam adequadamente as alturas. CARDOSO et al. (1989) e BARROS et al. (2002) trabalhando com a modelagem de altura para algumas espécies de Pinus encontraram resultados similares, os quais associaram a fatores que influenciam na relação hipsométrica, tais como sítio, idade, densidade e práticas silviculturais aplicadas no plantio. No que diz respeito à distribuição de resíduos, observa-se ausência de tendências na estimativa da altura e uma dispersão homogênea dos resíduos, com a amplitude de erro de $\pm 25 \%$ (Figura 1 ). 
Tabela 4. Coeficientes das equações ajustadas e resultados das estatísticas de ajuste para relação hipsométrica do melhor modelo para cada espécie.

Table 4. Coefficients of the adjusted equations and results of the adjusted statistics for the hypsometric relation of the best model for each species.

\begin{tabular}{|c|c|c|c|c|c|c|c|c|}
\hline \multirow{2}{*}{ Espécie } & \multirow{2}{*}{ № } & \multirow{2}{*}{ Autor } & \multicolumn{2}{|c|}{ Coeficientes } & \multirow{2}{*}{$\mathrm{R}^{2}{ }_{\mathrm{aj}}$} & \multirow{2}{*}{$\begin{array}{c}S_{y \cdot x} \\
(m)\end{array}$} & \multirow{2}{*}{$\begin{array}{c}S_{y \cdot x} \\
\%\end{array}$} & \multirow{2}{*}{$\mathrm{F}$} \\
\hline & & & $\beta_{0}$ & $\beta_{1}$ & & & & \\
\hline Pinus taeda & 6 & Henricksen & 0,515 & 5,915 & 0,355 & 1,2 & 6,4 & 28,62 \\
\hline Pinus elliottii & 9 & Linear Simples & 15,128 & 0,145 & 0,144 & 1,4 & 7,7 & 7,377 \\
\hline Pinus greggii & 4 & Naslund & 2,733 & 0,045 & 0,280 & 1,1 & 5,2 & 141,2 \\
\hline Pinus patula & 7 & Assmann & 26,425 & $-95,177$ & 0,295 & 1,1 & 4,8 & 23,64 \\
\hline
\end{tabular}

Em que: $\beta_{0}$ e $\beta_{1}$ : coeficientes ajustados dos modelos; $\mathrm{R}^{2}$ aj: coeficiente de determinação ajustado; $\mathrm{S}_{\mathrm{y} . \mathrm{x}(\mathrm{m}) \text { : erro padrão }}$ de estimativa em metros; $S_{y . x}(\%)$ : erro padrão da estimativa em percentual; $F$ : Teste $F$ de significância.
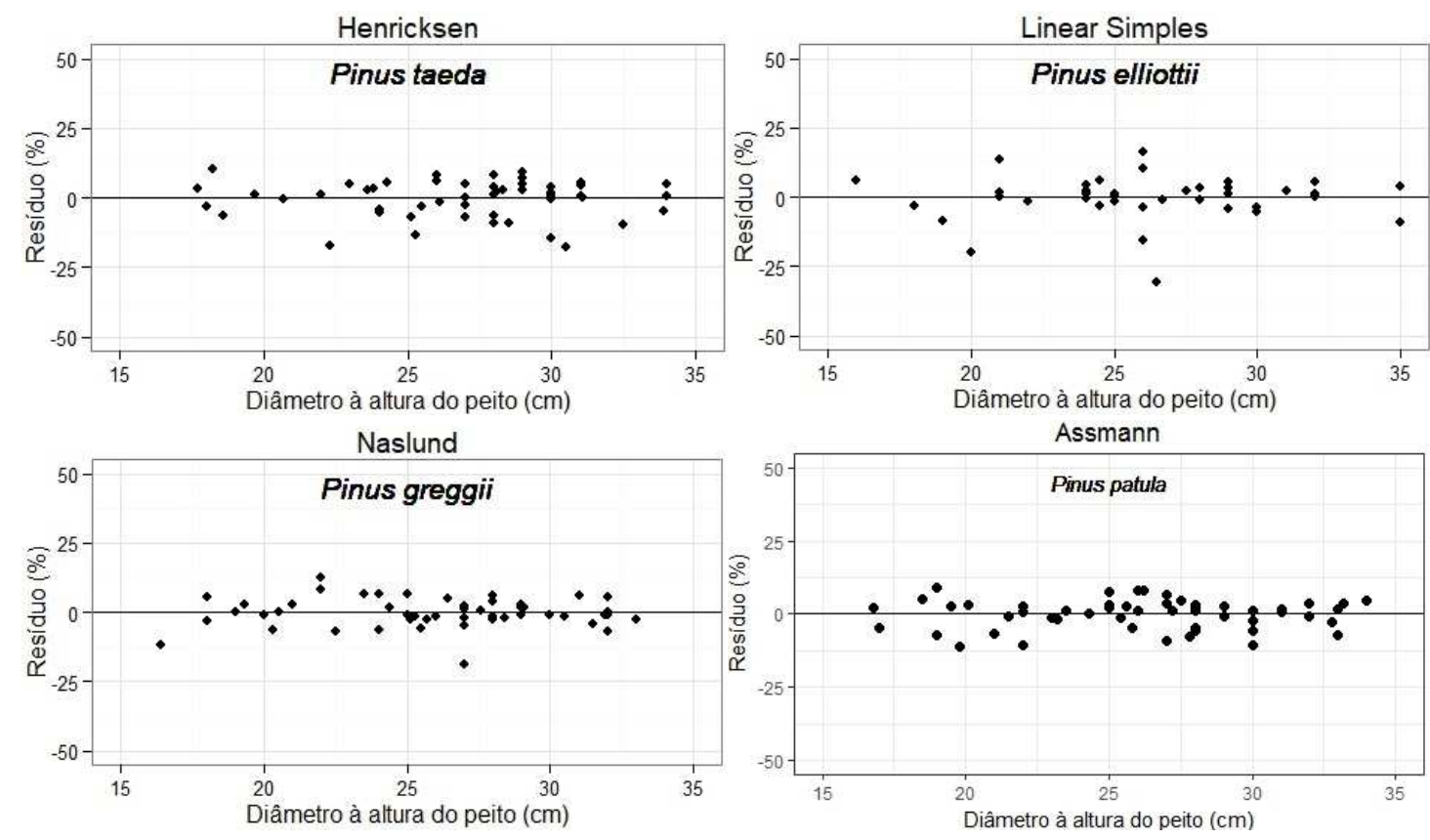

Figura 1. Distribuição gráfica de resíduos para os modelos de relação hipsométrica testados.

Figure 1. Graphic distribution of residuals for the tested hypsometric relation models.

\section{Volume}

As equações volumétricas para as espécies estudas apresentaram os coeficientes de determinação ajustado superiores a 0,97, com exceção da espécie Pinus patula que foi 0,91. Quanto ao erro padrão da estimativa $\left(S_{y . x} \%\right.$ apresentou valores baixos, menores que 10\%, variando entre 9,58 \% a 5,60 \% (Tabela 5).

Tabela 5. Melhores modelos e estatísticas para relações volumétricas para quatro espécies do gênero Pinus.

Table 5. Best models and statistics for volumetric relations for four species of the genus Pinus.

\begin{tabular}{lllllll}
\hline № & Espécie & Autor & $\mathrm{R}^{2} \mathrm{aj}_{\text {. }}$ & $\mathrm{S}_{\mathrm{yx}}\left(\mathrm{m}^{3}\right)$ & $\mathrm{S}_{\mathrm{yx}}(\%)$ & $\mathrm{F}$ \\
\hline 7 & Pinus taeda & Naslund & 0,978 & 0,026 & 5,6 & 933,14 \\
1 & Pinus elliottii & Spurr I & 0,970 & 0,029 & 6,8 & 616,96 \\
8 & Pinus greggii & Naslund modificado & 0,971 & 0,033 & 6,6 & 682,17 \\
3 & Pinus patula & Spurr II & 0,917 & 0,044 & 9,6 & 223,88 \\
\hline
\end{tabular}

Em que: $R^{2}$ aj: coeficiente de determinação ajustado; $S_{y x}\left(m^{3}\right)$ : erro padrão de estimativa em metro cubico; $S_{y x}(\%)$ : erro padrão da estimativa em percentual; F: Teste F de significância.

Existe um consenso por parte dos pesquisadores no meio florestal, que ao ajustar modelos para obtenção do volume, valores de coeficiente de determinação ajustados $\left(R^{2}{ }_{a j}\right)$ superiores a 0,95 e erro 
padrão da estimativa em porcentagem inferior $\left(S_{y . x \%}\right)$ a $10 \%$, são considerados adequados, porém quanto maior o $R^{2}$ e menor o $S_{y . x \%}$ maior a eficiência e a adequabilidade do modelo (THOMAS et al. 2006).

SILVESTRE et al. (2014) trabalhando com modelagem do volume para um povoamento de $P$. taeda no município de Lages, identificaram que o melhor modelo para estimar o volume foi o de Prodan, mas vale ressaltar que o modelo de Naslund apresentou resultados semelhantes ao obtidos no presente estudo, com $\mathrm{R}^{2}$ ajustado de 0,98 e erro padrão da estimativa de $11,6 \%$.

A análise residual mostra que a estimativa não apresentou qualquer viés relevante para nenhuma das espécies $( \pm 20 \%)$, proporcionando assim uma estimativa satisfatória quando a variável independente é mantida dentro dos intervalos dos dados utilizados para estimar os parâmetros do modelo, sendo, diâmetro à altura do peito de $15 \mathrm{a} 35 \mathrm{~cm}$ (Figura 2).

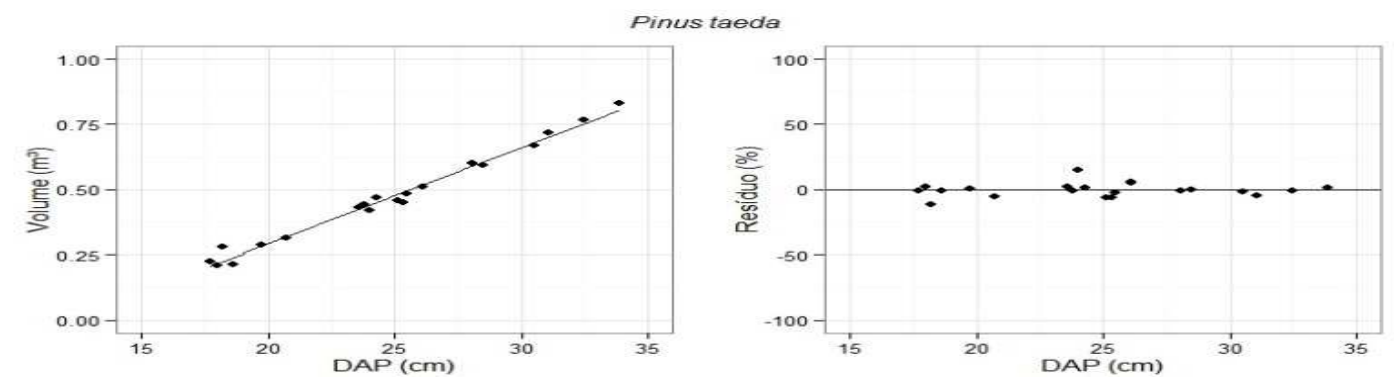

$\left.v_{i}=-0,640+0,001(\mathrm{~d})^{2}-\underset{\substack{0,00002 \\ \text { Pinus elliottii }}}{0} \mathrm{~d}^{2} \mathrm{~h}_{\mathrm{i}}\right)+\varepsilon_{\mathrm{i}}$
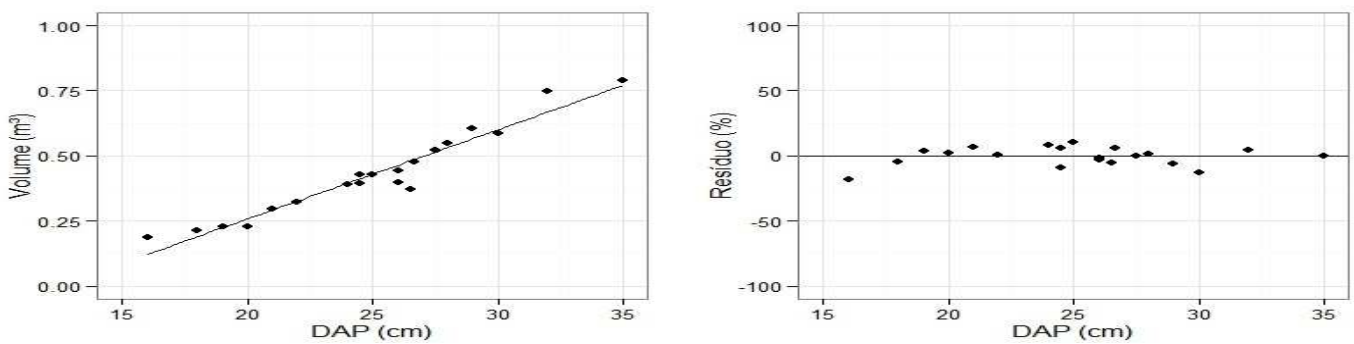

$v_{i}=0,027+0,00003\left(\mathrm{~d}^{2} \mathrm{~h}_{t}\right)+\varepsilon_{\mathrm{i}}$

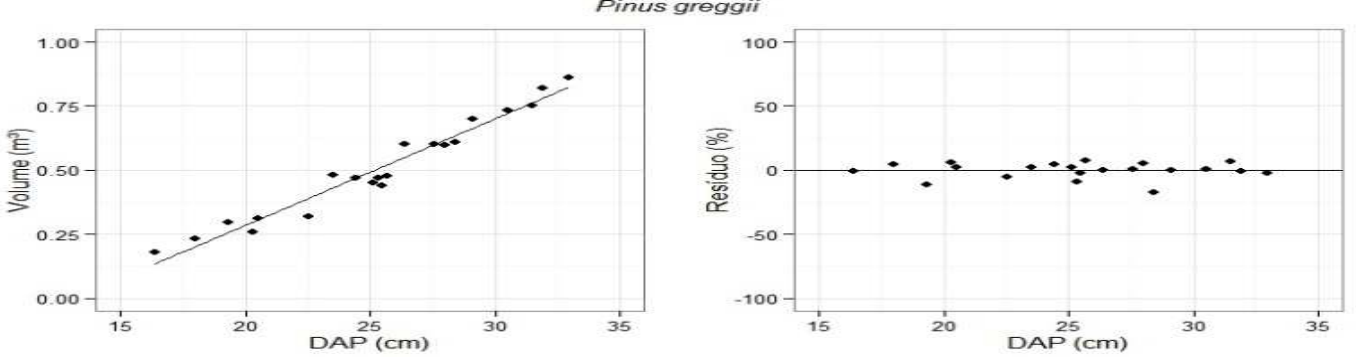

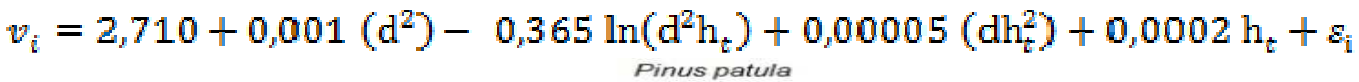
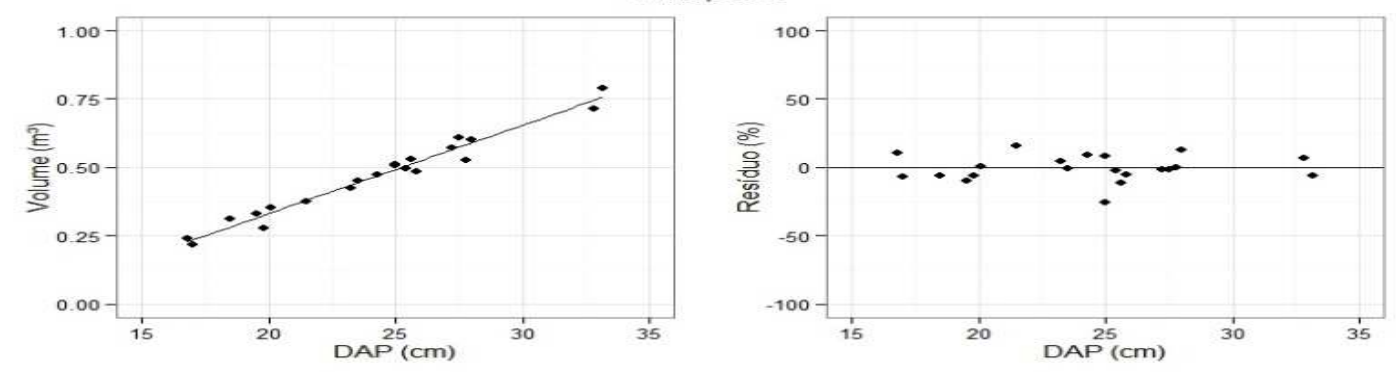

$v_{i}=-0,191+0,001\left(\mathrm{dh}_{\tau}\right)+\varepsilon_{\mathrm{i}}$

Figura 2. Volume individual observado com curva de regressão (linha) para o melhor modelo em cada espécie e resíduos estimados. Equação ajustada apresentada abaixo, em que: $v_{i}=$ volume individual $\left(\mathrm{m}^{3}\right) ; \mathrm{In}=$ logaritmo neperiano; $\mathrm{d}=$ diâmetro medido à $1,3 \mathrm{~m}$ do solo $(\mathrm{cm}) ; \mathrm{h}_{\mathrm{t}}=$ altura do fuste $(m)$ e; $\varepsilon_{\mathrm{i}}=$ erro aleatório.

Figure 2. Individual volume observed with (linear) regression curve for the best model in each species and estimated residuals. Adjusted equation presented below, where: $v_{i}=$ individual volume $\left(\mathrm{m}^{3}\right), \ln =$ Napierian logarithm, $D=$ diameter measured at $1.3 \mathrm{~m}$ above the soil $(\mathrm{cm}), \mathrm{h}_{\mathrm{t}}=$ stem height $(\mathrm{m})$, and $\varepsilon_{\mathrm{i}}=$ random error. 


\section{Funções de afilamento}

A equação que melhor descreveu o perfil do fuste das espécies foi a de Hradetzky, sendo que sua precisão foi maior para a espécie $P$. elliottii, com coeficientes de determinação ajustado de 0,98 e erro padrão da estimativa $\left(S_{y \cdot x} \%\right.$ de $5,33 \%$ (Tabela 6$)$.

Tabela 6. Estatísticas do modelo Hradetzky para estimar o diâmetro ao longo do fuste para quatro espécies de Pinus.

Table 6. Statistics from the Hradetzky model in order to estimate the diameter along the stem for four species of Pinus.

\begin{tabular}{|c|c|c|c|c|c|c|c|c|c|}
\hline \multirow{2}{*}{ Espécie } & \multicolumn{6}{|c|}{ Coeficientes } & \multirow[t]{2}{*}{$\mathrm{R}^{2}{ }_{\text {aj. }}$} & \multirow[t]{2}{*}{$\mathrm{S}_{\mathrm{y} \cdot \mathrm{x}(\mathrm{cm})}$} & \multirow[t]{2}{*}{$S_{y . x(\%)}$} \\
\hline & $\beta_{0}$ & $\beta_{1}$ & $\beta_{2}$ & $\beta_{3}$ & $\beta_{4}$ & $\beta_{5}$ & & & \\
\hline \multirow{2}{*}{ Pinus taeda } & 1,317 & 1,942 & $-1,424$ & $-0,054$ & $-1,843$ & 0,292 & 0,97 & 1,47 & 7,78 \\
\hline & Potências: & 0,9 & 2 & 3 & 0.5 & 25 & & & \\
\hline \multirow{2}{*}{ Pinus elliottii } & 15,139 & $-0,116$ & $-0,450$ & $-4,776$ & $-14,327$ & 10,014 & 0,98 & 1,05 & 5,33 \\
\hline & Potências: & 0,9 & 2 & 10 & 0,005 & 25 & & & \\
\hline \multirow{2}{*}{ Pinus greggii } & 1,413 & $-0,281$ & $-0,603$ & $-3,699$ & 3,312 & $-0,301$ & 0,98 & 1,46 & 7,61 \\
\hline & Potências: & 0,9 & 2 & 0,4 & 0,5 & 25 & & & \\
\hline \multirow{2}{*}{ Pinus patula } & 26,364 & 0,158 & $-0,564$ & $-1,625$ & $-25,745$ & 2,611 & 0,96 & 1,55 & 8,15 \\
\hline & Potências: & 0,9 & 2 & 10 & 0,005 & 25 & & & \\
\hline
\end{tabular}

Em que: Potências: Potências utilizadas no modelo ajustado.

Segundo STEPKA et al. (2017) diversos trabalhos foram desenvolvidos com intuito de modelar a forma e estimar o diâmetro ao longo do fuste para espécies do Pinus. TEO et al. (2013) ao realizarem estimativas de diâmetros em diferentes alturas para $P$. elliotti, na Região de Caçador, SC, identificaram que o modelo de Hradetzky apresentou melhor desempenho para estimar o diâmetro ao longo do fuste dos indivíduos, para as idades de 10, 18 e 27 anos. YOSHITANI JUNIOR et al. (2012) ao avaliarem funções de afilamento para estimar diâmetros e volumes ao longo do fuste de $P$. taeda, identificaram os modelos de Hradezky e de Schöepfer como os melhores devido apresentarem resultados satisfatórios, com alto valor de coeficiente de determinação ajustado e baixos valores de erro padrão da estimativa.

ASSIS et al. (2002) ao avaliarem a estimativa de diâmetros ao longo do fuste para $P$. taeda utilizando modelos polinomiais não segmentados, na Região de Jaguariaíva, identificaram que os modelos de Hradetzky e Goulding e Murray apresentaram estimativas mais acuradas dos diâmetros ao longo do fuste. YOSHITANI JUNIOR et al. (2012) identificaram o modelo Hradetzky como o melhor para estimar o diâmetro ao longo do fuste para $P$. taeda, o mesmo apresentou $r^{2}$ ajustado de $98,2 \%$ e $S_{y . x \%}$ de $7,92 \%$, resultados semelhantes ao do presente estudo. KOEHLER et al. (2013) chegaram à conclusão que o modelo de Hradetzky é o melhor para descrever o perfil do fuste de $P$. taeda no município de Santa Catarina, o mesmo apresentou coeficiente de determinação ajustado de $99,7 \%$ e $S_{y . x \%} 7,57 \%$

Ao analisar a Figura 3 é possível observar que $o$ afilamento foi mais abrupto na base da árvore, entre $25-50 \%$ da altura total da árvore, a tendência tornou-se mais suave. Então, dos 50-100\%, o afilamento novamente aumentou isso ocorre para todas as espécies.

A partir do gráfico residual é possível inferir que a equação ajustada confere estimativas mais precisas acima de $10 \mathrm{~cm}$ de diâmetro. Embora o fato possa gerar argumentos para ineficiência da equação, é importante ressaltar que esses diâmetros são não comerciais, sendo menos importantes. Apesar das análises dos parâmetros de qualidade e precisão dos ajustes fornecerem importantes requisitos para a escolha do melhor modelo, estas de forma isolada não garantem uma conclusão satisfatória sobre o desempenho deles, pois podem ocorrer erros grosseiros em determinados cenários que não são detectados pelos parâmetros de precisão, sendo assim necessário realizar a análise da distribuição gráfica dos resíduos (MIRANDA et al. 2014, STEPKA et al. 2017). 
Pinus taeda
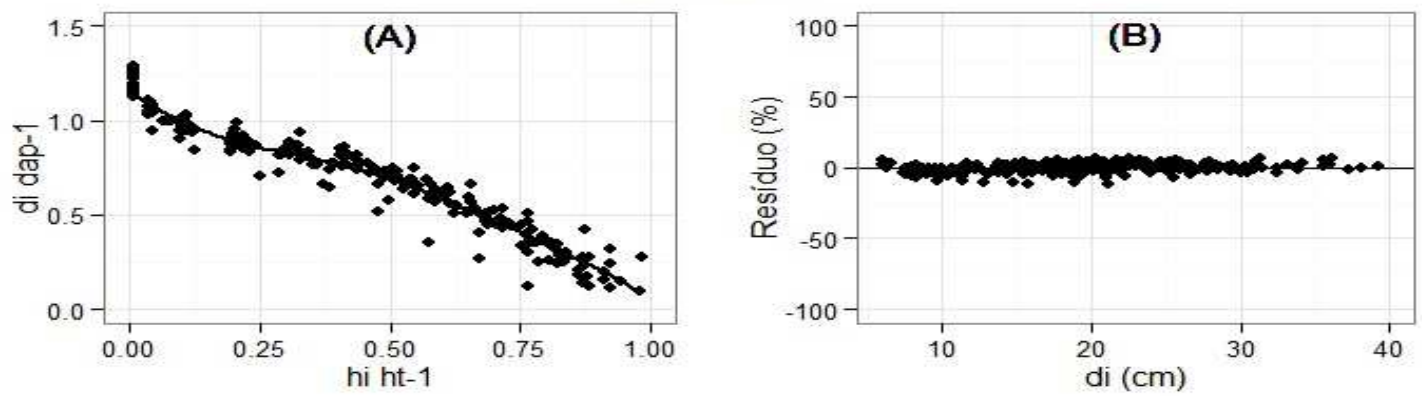

Pinus elliottii
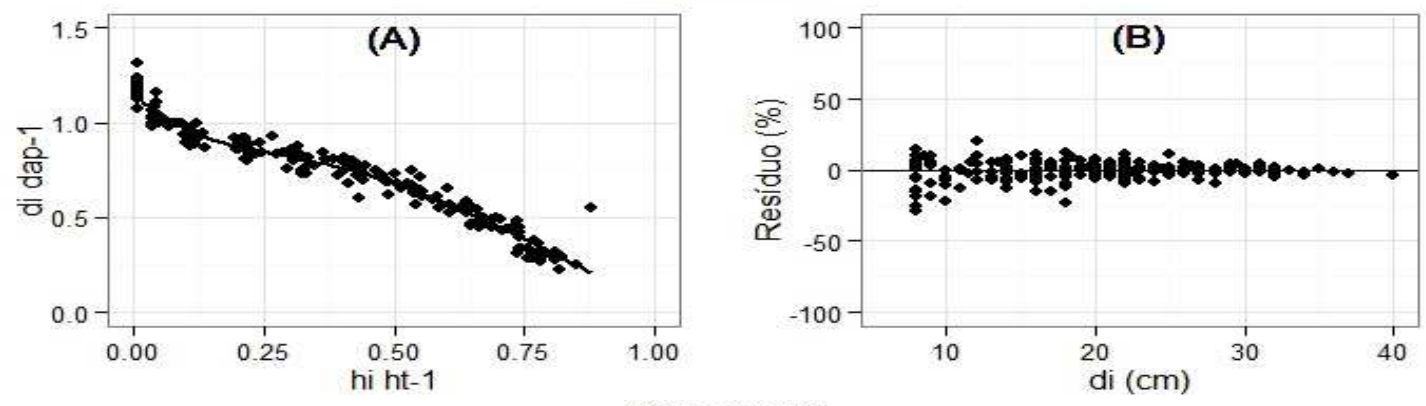

Pinus greggii
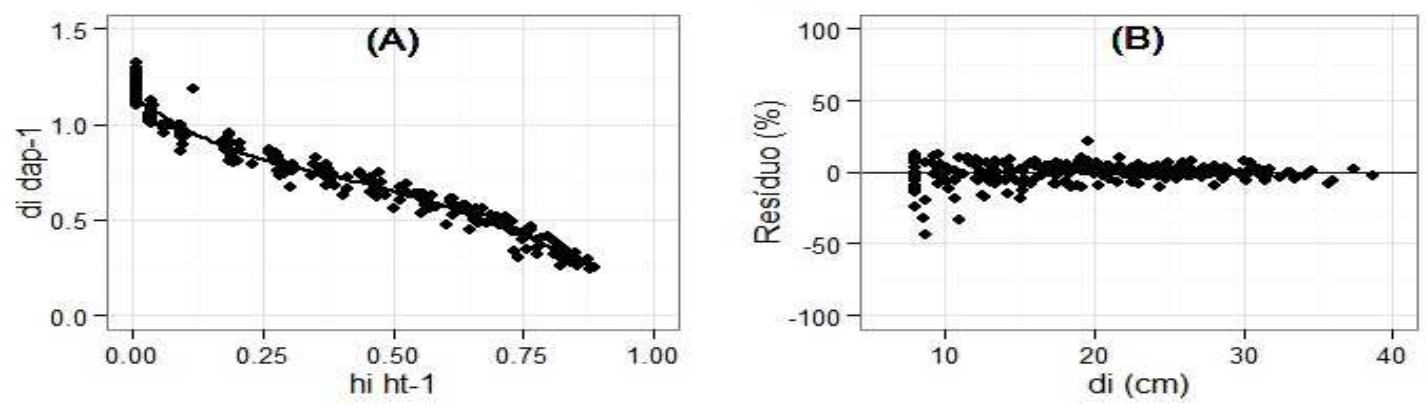

Pinus patula
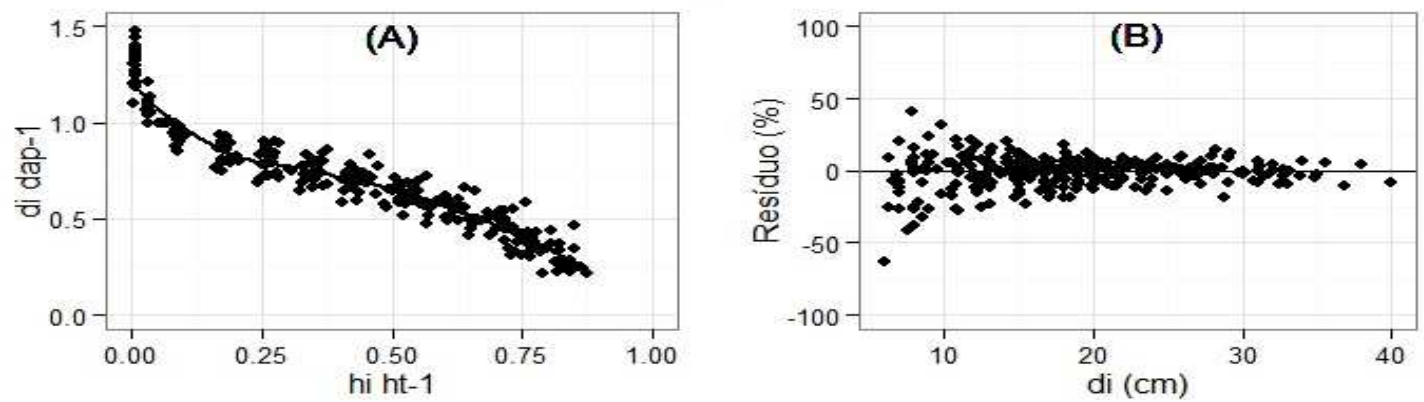

Figura 3. Perfil do fuste dos indivíduos de Pinus (A) e distribuição gráfica de resíduos para o modelo de afilamento de Hradetzky ao estimar o diâmetro em diferentes partes do fuste (B).

Figure 3. Stem profile of individuals of Pinus $(A)$ and graphic distribution of residuals for the Hradetzky taper model used to estimate the diameter in different parts of the stem (B).

\section{CONCLUSÃO}

Os melhores modelos para a relação hipsométrica das espécies foram Henricksen ( $P$. taeda), linear simples ( $P$. elliottii), Naslund ( $P$. greggii) e Assmann ( $P$. patula). Esses modelos apresentaram valores baixos de coeficientes de determinação ajustado, porém, o erro padrão da estimativa pode ser considerado satisfatórios para as equações.

Os modelos de Naslund, Spurr I, Naslund modificado e Spurr II, apresentaram-se como os melhores modelos para estimar a volumetria das espécies $P$. taeda, $P$. elliottii, $P$. greggii, $P$. patula respectivamente na área de estudo;

O modelo não segmentado de Hradetzky apresentou as melhores estatísticas entre as funções de afilamento na estimativa dos diâmetros ao longo do fuste para todas as espécies estudadas. 


\section{REFERÊNCIAS}

ABREU ECR. 2000. Modelagem para prognose precoce do volume por classe diamétrica para Eucalyptus grandis. Dissertação (Mestrado em Engenharia Florestal). Lavras: UFL. 69p.

AGUIAR AV et al. 2011. Programa de melhoramento de Pinus da Embrapa Florestas. Colombo: Embrapa florestas. (Documentos 233).

ALVARES CA et al. 2013. Köppen's climate classification map for Brazil. Meteorologische Zeitschrift, Stuttgart 22: 711 728.

ARAÚJO EJG et al. 2012. Relações dendrométricas em fragmentos de povoamentos de Pinus em Minas Gerais. Pesquisa Florestal Brasileira 32: 355-366.

ASSIS AL et al. 2002. Comparação de modelos polinomiais segmentados e não-segmentados na estimativa de diâmetros e volumes ao longo do fuste de Pinus taeda. Cerne 7: 20-42.

ATANAZIO KA et al. 2017. Comparação de modelos para relação hipsométrica em floresta de Pinus taeda L. no município de Enéas Marques, Paraná. Revista Scientia Agraria Paranaensis 16: 535-541.

BARROS DA et al. 2002. Comportamento de modelos hipsométricos tradicionais e genéricos para plantações de Pinus oocarpa em diferentes tratamentos. Boletim de Pesquisa Florestal 45: 3-28.

CARDOSO DJ et al. 1989. Avaliação da influência dos fatores idade e sítio na relação hipsométrica para Pinus taeda nas regiões central e sudoeste do estado do Paraná. Revista Floresta 19: 96-115.

COELHO VCM. 2010. Avaliação do manejo da produção econômica de madeira de Pinus taeda L. com características qualitativas superiores. Dissertação (Mestrado em Engenharia Florestal) Curitiba: UFPR. 121p.

COSTA LPE. 2008. Relações biométricas em povoamentos jovens de Pinus taeda L. na província de Corrientes de República Argentina. Tese (Doutorado em Manejo Florestal). Santa Maria: UFSM. 128p.

COSTA EA et al. 2016. Função de afilamento e sortimento de madeira para Araucaria angustifolia. Revista Ciência Florestal 26: 523-533.

DONADONI AX et al. 2010. Relação hipsométrica para Pinus caribaea var. hondurensis e Pinus tecunumanii em povoamento homogêneo no Estado de Rondônia. Ciência Rural 40: 2499-2504.

GARCIA SLR et al. 1993. Análise do perfil do tronco de morototó (Didymoopanax morototonii) em função do espaçamento. In: Congresso Florestal Panamericano e Congresso Florestal Brasileiro. Resumos... Curitiba: SBS e SBEF. p.485-491.

HRADETZKY J. 1976. Analyse und interpretation statistisher abränger keisen. (Biometrische Beiträge zu aktuellen forschuns projekten). Baden: Württemberg Mitteilungen der FVA. p.146.

IBA. 2019. Indústria Brasileira de Árvores. Relatório IBÁ - 2018. São Paulo: IBA.

LEITE HG et al. 2006. Função de afilamento para Virola surinamensis (ROLL.) Warb. Revista Árvore 30: 99-106.

KOEHLER SV et al. 2013. Modelos de afilamento para Pinus taeda por classes de idade. Floresta e Ambiente 20: 470 479.

KOZAK A et al.1969. Taper functions and their application in forest inventory. Forestry Chronicle 45: 278-283.

MACHADO SA et al. 2002. Modelagem do volume individual para diferentes idades e regimes de desbaste em plantações de Pinus oocarpa. Revista Ciências Exatas e Naturais 4: 186-197.

MIRANDA DLC et al. 2014. Estimativa do volume em árvores de Hymenaea coubaril L. e Trattinnickia bursefolia Mart. no norte de Mato Grosso. Nativa 2: 219- 223.

NICOLETTI MF. 2017. Modelagem não linear mista e método bivariado para sortimento da produção de Pinus taeda L. Tese (Doutorado em Engenharia Florestal). Curitiba: UFPR. 132p.

RUFINI AL et al. 2010. Equações volumétricas para o cerrado Sensu stricto, em Minas Gerais. Cerne 16: 1-11.

SCHÖEPFER W. 1966. Autimatisierung des massen, sorten and wertberechnung stenender waaldbestande schriftenreihe bad. Wurtt-Forstl.

SILVESTRE R et al. 2014. Equações volumétricas em povoamentos de Pinus taeda L. no município de Lages-SC. Revista Nativa 2: 1-5.

SOARES CPB et al. 2011. Equações hipsométricas, volumétricas e de Taper para onze espécies nativas. Revista Árvore 35: 1039-1051.

STEPKA TF et al. 2017. Modelos volumétricos e funções de afilamento para Pinus taeda L. na Região dos Campos Gerais, Paraná, Brasil. Revista Espacios 38: 1-9.

TEO SJ et al. 2013. Modelos de afilamento para Pinus elliottii em diferentes idades, na Região de Caçador, SC. Floresta 43: 439-452.

THIERSCH A. 1997. A eficiência das distribuições diamétricas para prognose da produção de Eucalyptus camaldulensis. Dissertação (Mestrado em Engenharia Florestal). Lavras: UFL. 155p.

THIERSCH CR et al. 2013. Estimativa da relação hipsométrica em clones de Eucalyptus sp. com o modelo de Curtis ajustado por métodos bayesianos empíricos. Revista Árvore 1: 1-8.

THOMAS C et al. 2006. Comparação de equações volumétricas ajustadas com dados de cubagem e análise de tronco. Ciência Florestal 16: 319-327.

YOSHITANI JUNIOR M et al. 2012. Funções de afilamento para plantios desbastados de Pinus taeda. Floresta 42: 169176. 